\title{
Hot-electron spectrometry with quantum point contacts
}

\author{
J. G. Williamson, H. van Houten, C. W. J. Beenakker, \\ M. E. I. Broekaart, and L. I. A. Spendeler* \\ Philips Research Laboratories, NL-5600 JA Eindhoven, The Netherlands \\ B. J. van Wees \\ Department of Applied Physics, Delft University of Technology, NL-2600 GA Delft, The Netherlands \\ C. T. Foxon \\ Philips Research Laboratories, Cross Oak Lane, Redhill, Surrey RHI 5HA, United Kingdom
}

(Received 17 July 1989)

\begin{abstract}
Ballistic transport of electrons over several micrometers with excess energy of up to the order of the Fermi energy has been observed in the two-dimensional electron gas of a $\mathrm{GaAs}_{\mathrm{Al}} \mathrm{Al}_{x} \mathrm{Ga}_{1-{ }_{-}} \mathrm{As}$ heterostructure. Quantum point contacts in an electron-focusing geometry have been used as a novel magnetic spectrometer to measure directly the kinetic energy of injected electrons. The observed energy gain is linear in the total applied voltage, and the slope allows a determination of the local voltage drop over the point contact.
\end{abstract}

A number of striking new transport effects have recently been reported in the quantum ballistic regime, in a two-dimensional electron gas (2D EG) in a GaAs- $\mathrm{Al}_{x} \mathrm{Ga}_{1-x}$ As heterostructure. ${ }^{1-5}$ In particular, electron focusing ${ }^{6}$ by means of a magnetic field in a $2 \mathrm{D}$ EG was reported. ${ }^{7-9}$ In this experiment two adjacent quantum point contacts were used as injector and collector of ballistic electrons. Focusing peaks in the collector voltage $V_{c}$ were observed at magnetic field values corresponding to an integral number of cyclotron orbit diameters between injector and collector. The experiments were carried out at low injection voltages (a few $\mu \mathrm{V}$ ) so that the excess energy of the injected electrons was much smaller than the Fermi energy $E_{F}(\approx 14 \mathrm{meV})$.

Hot-electron spectroscopy in semiconductors was pioneered in vertical-transport structures ${ }^{10}$ and has been extended recently to the lateral ballistic transport regime. ${ }^{11}$ In these experiments the energy was measured by varying the height of a collector barrier. Here we present a novel magnetic spectrometer technique, based on electron focusing, where the energy is extracted from the cyclotron radius of the injected hot electrons in a region local to a quantum point contact. Nonequilibrium electron focusing has previously been studied in metals ${ }^{12-14}$ where $E_{F}$, typically a few $\mathrm{eV}$, is much larger than typical applied voltages, so that the relative increase in energy could be neglected. An injector current dependence of the position of the focusing peaks in these experiments has been attributed to the magnetic field induced by the current itself. ${ }^{12,15}$

The electron-focusing device $e^{7,8}$ consists of two adjacent point contacts of variable width (inset in Fig. 1), defined electrostatically by means of split Schottky gates in the 2D EG of a GaAs- $\mathrm{Al}_{x} \mathrm{Ga}_{1-x}$ As heterostructure. The electron density was $4.0 \times 10^{15} \mathrm{~m}^{-2}, E_{F}=14.2 \mathrm{meV}$, and the transport mean free path was $9 \mu \mathrm{m}$. The pointcontact spacing was nominally $1.5 \mu \mathrm{m}$. We distinguish three regions: region $i$ behind the injector point contact, region $c$ behind the collector, and the wide twodimensional electron gas region $s$ (where the electron focusing takes place). A dc bias voltage $V_{\mathrm{dc}}$ of a few $\mathrm{mV}$ (Ref. 16) and an ac voltage of $100 \mu \mathrm{V}$ were applied between terminals 1 and 2 (the injector point contact). We obtain a differential resistance $d V_{c} / d I_{t}$ from the ac voltage across the collector (terminals 3 and 4) normalized to the ac injector current.

We have measured $d V_{c} / d I_{1}$ as a function of a perpendicular magnetic field $B$ for different $V_{\text {dc }}$ (at a nominal temperature of $100 \mathrm{mK}$ ). At zero bias focusing occurs whenever the point-contact spacing $L$ is an integral multiple of the cyclotron diameter $\left(8 m E_{F}\right)^{1 / 2} / e B$. This leads to focusing peaks at $B=n B_{\text {focus }}, n=1,2,3 \ldots$, with

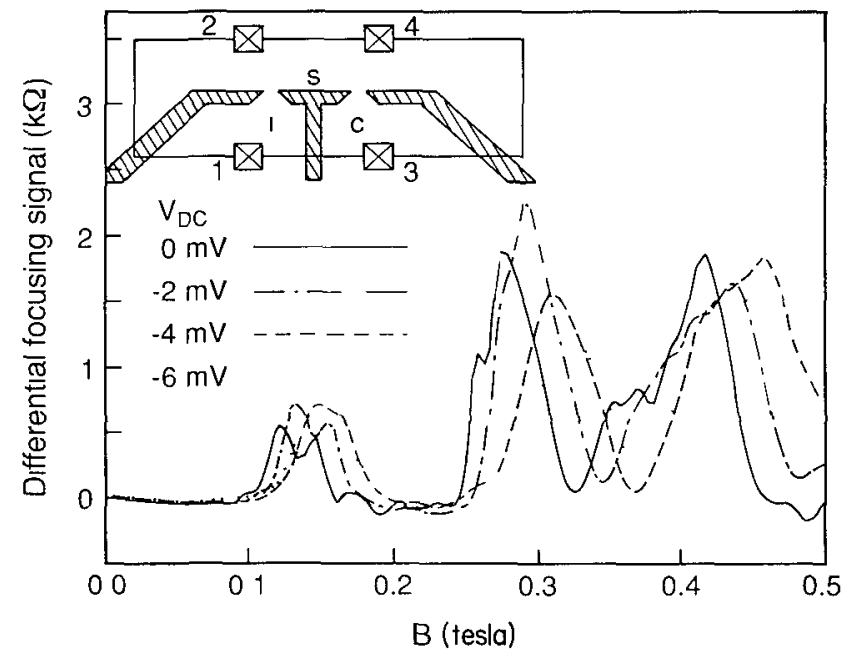

FIG. 1. Electron-focusing spectra $d V_{34} / d I_{12}$ for various applied dc bias voltages. Inset: schematic device diagram. The shaded parts indicate the gate used to define the point contacts and the 2D EG boundary, and the squares denote the Ohmic contacts. 


$$
B_{\text {focus }}=\left(8 m E_{\text {focus }}\right)^{1 / 2} / e L
$$

and $E_{\mathrm{focus}}=E_{F}$. We will see that, for a differential resistance measurement Eq. (1) still holds for a wide range of voltage drops $V$ over the injector point contact, with $E_{\text {focus }}=E_{F}-e V$. In Fig. 1 we show the evolution of the focusing spectra with increasing negative bias voltage. At zero bias, both the injector and collector resistances were quantized at $h / 2 e^{2} \approx 13 \mathrm{k} \Omega$ (corresponding to a single occupied $1 \mathrm{D}$ subband ${ }^{1,2}$ ). The general trend is clearly a shift of the focusing peaks to higher magnetic field, consistent with Eq. (1). Superimposed on the focusing peaks we see interference fine structure. ${ }^{7-9}$ Figure 2 shows $d V_{c} / d I_{t}$ for $V_{\mathrm{dc}}=0,-4$, and $+4 \mathrm{mV}$. The peaks shift in opposite directions for positive and negative $V_{\mathrm{dc}}$. Note also that the peak height for $V_{\mathrm{dc}}=+4 \mathrm{mV}$ is considerably smaller than that for $-4 \mathrm{mV}$.

These results can be understood in terms of a simple model. We assume that the electric field caused by the applied voltage is negligible outside the immediate vicinity of the injector point contact. We assume adiabatic transport, i.e., no intersubband scattering, and we consider the case of a single occupied subband in the point contact. We define local Fermi energies $E_{F}^{s}, E_{F}^{l}=E_{F}^{s}-e V$, and $E_{F}^{c}$ for each region. Here $-e$ is the electron charge, and $V$ is the voltage drop over the injector point contact, which may be smaller than the total bias voltage $V_{\mathrm{dc}}$ (e.g., because of the background resistance associated with the Ohmic contacts). Let $E_{1}$ be the energy of the bottom of the lowest subband evaluated at the "bottleneck" of the injector where it is maximal. ${ }^{17}$ Note that $E_{1}$ will depend on $V$. Following Ref. 17 we can calculate the current $J(E) d E$ carried by electrons in the lowest subband with energies between $E$ and $E+d E$. At the bottleneck the states moving from $i$ to $s$ are filled from $E_{1}$ to $E_{F}^{l}$ (provided that $E_{F}^{l}>E_{1}$ ), and the states moving from $s$ to $i$ are filled from $E_{1}$ to $E_{F}^{s}$ (provided that $\left.E_{F}^{S}>E_{1}\right)$. The current density is nonzero only if $E>E_{1}$ and $\min \left\{E_{F}, E_{F}^{s}\right\}<E<\max \left\{E_{F}^{l}, E_{F}^{s}\right\}$, in which case $J(E)=2 e / h$, independent of $E$ (cf. Ref. 17). This gives [with $\Theta(x)=1$ for $x>0$ and $\Theta(x)=0$ for $x<0$ ]

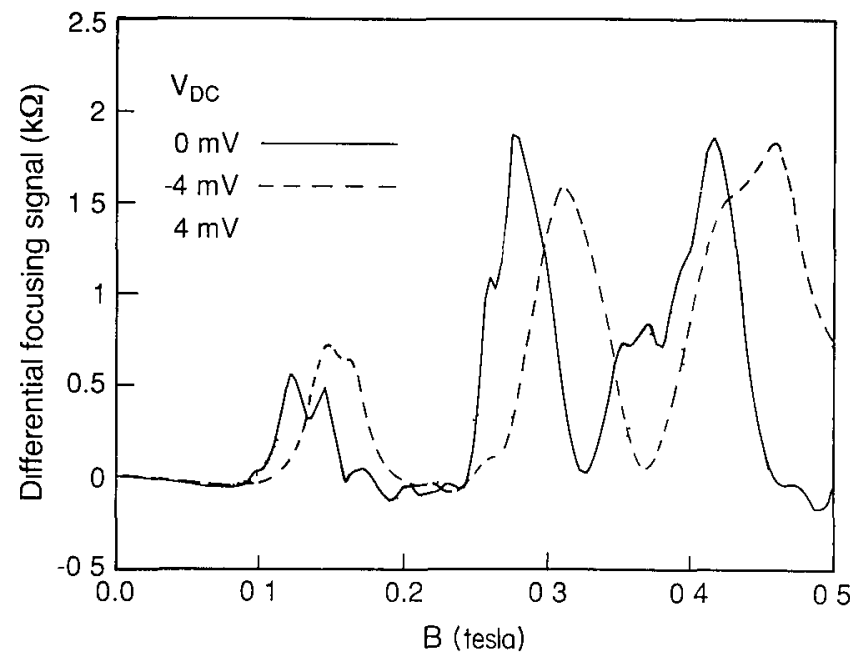

FIG. 2. Electron-focusing spectra for dc bias voltages of the opposite sign.

$$
J(E)=\left\{\begin{array}{l}
(2 e / h) \Theta\left(E_{F}^{\varsigma}-e V-E\right) \Theta\left(E-E_{1}\right) \Theta\left(E-E_{F}^{\varsigma}\right) \\
\text { for } V<0 \\
(2 e / h) \Theta\left(E+e V-E_{\Gamma}^{s}\right) \Theta\left(E-E_{1}\right) \Theta\left(E_{F}^{s}-E\right) \\
\text { for } V>0 .
\end{array}\right.
$$

Electrons are focused onto the collector after $n-1$ specular reflections at the boundary if $E=E_{\text {focus }}$ $=(L e B / n)^{2} / 8 m$ [cf. Eq. (1)]. We may thus write for the contribution of these focused electrons to the collector voltage $\delta V_{c}=$ const $\times J\left(E_{\text {focus }}\right)$. (We consider the case where $E_{1}$ is above the bottom of the lowest subband in the collector, so that no further energy selection occurs.) The undetermined constant prefactor will presumably vary smoothly with magnetic field and voltage, and its effect on the position of the focusing peaks is ignored. A differential measurement gives $d V_{c} / d I_{t}$ $=\left(d V_{c} / d V\right) \quad\left(d V / d I_{1}\right) . \quad$ The second factor is the differential resistance of the injector, which varies smoothly ${ }^{17}$ with $B$ and $V$. The first factor determines the position of the peaks in the differential resistance. The contribution from $\delta V_{c}$ to this factor is

$$
\frac{d \delta V_{c}}{d V}=\left\{\begin{aligned}
\text { const } \times\left[\delta ( E _ { F } ^ { s } - e V - E _ { \mathrm { focus } } ) \Theta \left(E_{\mathrm{focus}}\right.\right. & \left.-E_{1}\right) \Theta\left(E_{\mathrm{focus}}-E_{F}^{s}\right) \\
& \left.-\alpha \Theta\left(E_{F}^{s}-e V-E_{\mathrm{focus}}\right) \delta\left(E_{\mathrm{focus}}-E_{1}\right) \Theta\left(E_{\mathrm{focus}}-E_{F}^{s}\right)\right], \quad \text { for } V<0 \\
\operatorname{const} \times\left[\delta ( E _ { \mathrm { focus } } + e V - E _ { F } ^ { s } ) \Theta \left(E_{\mathrm{focus}}\right.\right. & \left.-E_{1}\right) \Theta\left(E_{F}^{s}-E_{\text {focus }}\right) \\
& \left.+\alpha \Theta\left(E_{\text {focus }}+e V-E_{F}^{s}\right) \delta\left(E_{\text {focus }}-E_{1}\right) \Theta\left(E_{F}^{s}-E_{\text {focus }}\right)\right] \text { for } V>0 .
\end{aligned}\right.
$$

The positive parameter $\alpha=-e^{-1} d E_{1} / d V$ describes the voltage dependence of the subband bottom (or barrier height) at the bottleneck of the injector. We see from Eq. (3a) that a peak occurs in $d V_{c} / d I_{l}$ at $B$ values such that $E_{\text {focus }}=E_{F}^{s}-e V$ for all negative $V$. In addition, a dip with an amplitude reduced by a factor $\alpha$ occurs at $E_{\text {focus }}=E_{1}$, provided that $E_{F}^{s}<E_{1}$, i.e., the Fermi level in the $2 \mathrm{D}$ EG region $s$ drops below the barrier energy.
For positive $V$, Eq. (3b) predicts only one peak at $E_{\text {focus }}=\max \left\{E_{1}, E_{\Gamma}^{\text {s }}-e V\right\}$. The peak should occur at $E_{1}$ if $E_{1}>E_{\Gamma}^{s}-e V=E_{\Gamma}^{\prime}$, in which case it will have an amplitude reduced by a factor $\alpha$.

Although the distribution of injected electrons extends over a wide range of energies, the differential technique selects primarily only those electrons with maximal or minimal injection energy. One can thus study the dy- 
namics of "hot" electrons for $V<0$ and of "cool" unoccupied electron states (holes in the conduction band) for $V>0$, with an energy resolution determined by the magnitude of the ac voltage.

In Fig. 3 we have plotted $E_{\text {focus }}$ obtained from the position of the $n=3$ focusing peak as a function of $V_{\mathrm{dc}}$. We first consider the region for $V_{\mathrm{dc}}$ between -8 and $+3 \mathrm{mV}$ where $E_{\text {focus }}$ is clearly linear in $V_{\mathrm{dc}}$. A linear leastsquares fit in this region (cf. the solid line in Fig. 3) yields

$$
E_{\text {focus }}=-0.68 e V_{\mathrm{dc}}+14.4 \mathrm{meV} \text {. }
$$

For $V_{\mathrm{dc}}=0$ the electron energy $E_{\text {focus }}$ is very close to $E_{F}$ measured using the Shubnikov-de Haas oscillations (14.2 $\mathrm{MeV}$; the horizontal dashed line in Fig. 3), showing that the focusing period can give an accurate determination of the Fermi energy. The slope of $E_{\text {focus }}$ versus $V_{\mathrm{dc}}$ yields the ratio of the local voltage drop over the point contact to the voltage drop over the entire sample. Thus the local energy gain on crossing the point contact is only $-0.68 \mathrm{eV}$ dc. The total resistance over which $V_{\mathrm{dc}}$ was applied was $19.4 \pm 0.3 \mathrm{k} \Omega$ including the $300 \Omega$ series resistor. So our measurements imply an injector pointcontact resistance of $13.2 \pm 0.3 \mathrm{k} \Omega$, in good agreement with the quantized point-contact resistance ${ }^{1,2}$ $h / 2 e^{2}=12.9 \mathrm{k} \Omega$. We stress that in obtaining this value, only the focusing peak spacing has been used, and not the absolute value of $d V_{c} / d I_{i}$. Also, we have effectively used the device itself, and not an external voltmeter, to measure the energy gain.

The deviation from linearity for $V_{\mathrm{dc}}>+3 \mathrm{mV}$ may arise from the effects discussed above when $E_{F}^{l}<E_{1}$. Additionally, the collector point contact may impose an additional energy selection on the electron-focusing signal. This will be important at high positive $V_{\text {dc }}$ (but not for negative $V_{\mathrm{dc}}$ ). These effects are not studied in detail here, as our main concern is the linear region at small biases. The reduction in peak height for positive voltages may be due to a combination of these effects and possibly also because of a higher scattering rates for cool "holes." The reduction in slope in Fig. 3 below $-8 \mathrm{mV}$ is presumably due to inelastic scattering processes in the point-contact region. For large negative $V_{\mathrm{dc}}$ such that $E_{F}^{s}<E_{1}$, one would also expect to see a small dip at $E_{\text {focus }}=E_{1}$, but

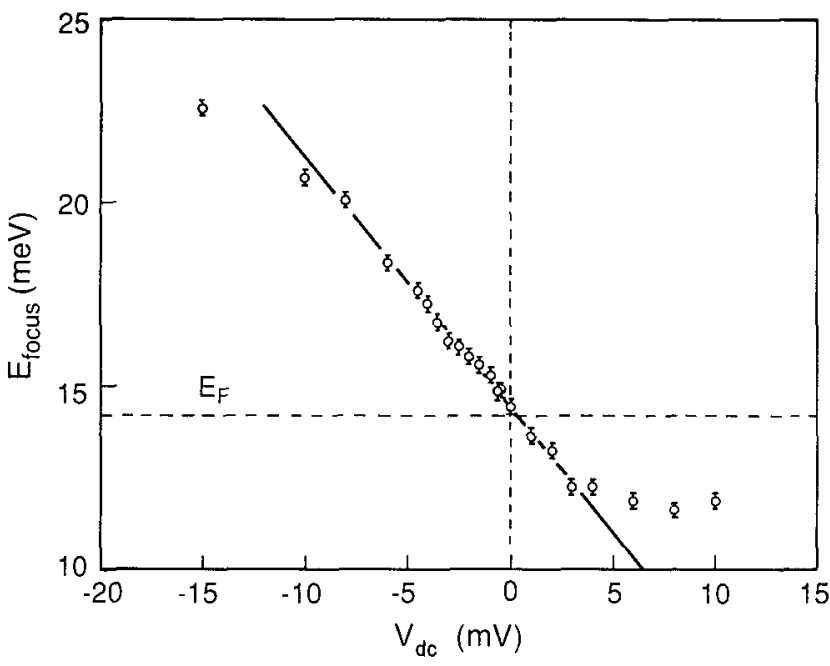

FIG. 3. Spectrometer energy $E_{\text {focus }}$ extracted from the focusing peak spacing as a function of applied dc bias voltage. The solid line has been obtained from a linear least-squares fit.

this has not been resolved experimentally.

In conclusion, we have measured the energy of hot electrons (and cold holes) in a region within a scattering length of a quantum point contact, where the motion is ballistic. Over a wide range of dc bias voltages, the energy is found to increase linearly in agreement with a simple model. The experiment demonstrates conclusively that ballistic transport of hot electrons, with energy $\approx 1.5 E_{F}$, in a two-dimensional electron gas can take place over distances larger than $\pi L / 2 \approx 2.3 \mu \mathrm{m}$. Very recently Sivan, Heiblum, and Umbach ${ }^{18}$ have performed a hot-electron experiment in a geometry with two point contacts in series, and found that the inelastic mean free path at energies below the longitudinal optical-phonon energy is an order of magnitude larger than theoretical predictions. Our experiment provides an independent confirmation of their result, using a quite different measurement technique. In addition we believe our experiment to be the first measurement of a local voltage drop in a nanostructure device. ${ }^{19}$

We thank M. F. H. Schuurmans for valuable discussions.
*Also at: Ecole de Physique de Grenoble Magistere, University Joseph Fourier, 38041 Grenoble CEDEX, France.

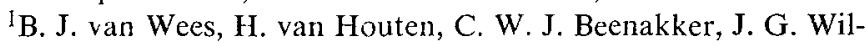
liamson, L. P. Kouwenhoven, D. van der Marel, and C. T. Foxon. Phys. Rev. Lett. 60, 848 (1988).

${ }^{2}$ D. A. Wharam, T. J. Thornton, R. Newbury, M. Pepper, H. Ahmed, J. E. F. Frost, D. G. Hasko, D. C. Peacock, D. A. Ritchie, and G. A. C. Jones, J. Phys. C 21, L209 (1988).

${ }^{3}$ M. L. Roukes, A. Scherer, S. J. Allen, Jr., H. G. Craighead, R. M. Ruthen, E. D. Bcebe, and J. P. Harbison, Phys. Rev. Lett. 59, 3011 (1987).

${ }^{4}$ G. Timp, A. M. Chang, J. E. Cunningham, T. Y. Chang, P. Mankiewich, R. Behringer, and R. E. Howard, Phys. Rev. Lett. 58, 2814 (1987).

${ }^{5}$ P. H. M. van Loosdrecht, C. W. J. Beenakker, H. van Houten, J. G. Williamson, B. J. van Wees, J. E. Mooij, C. T. Foxon, and J. J. Harris, Phys. Rev. B 38, 10162 (1988); B. J. van Wees, L. P. Kouwenhoven, C. J. P. M. Harmans, J. G. Williamson, C. E. Timmering, M. E. I. Broekaart, C. T. Foxon, and J. J. Harris, Phys. Rev. Lett. 62, 2523 (1989).

${ }^{6}$ V. S. Tsoi, Zh. Eksp. Teor. Fiz. 68, 984 (1975) [Sov. Phys.JETP 41, 927 (1976)].

${ }^{7}$ H. van Houten, B. J. van Wees, J. E. Mooij, C. W. J. Beenakker, J. G. Williamson, and C. T. Foxon, Europhys. Lett. 5, 721 (1988).

${ }^{8}$ H. van Houten, C. W. J. Beenakker, J. G. Williamson, M. E. I. Broekaart, P. H. M. van Loosdrecht, B. J. van Wees, J. E. Mooij, C. T. Foxon, and J. J. Harris, Phys. Rev. B 39, 8556 (1989).

${ }^{9}$ C. W. J. Beenakker, H. van Houten and B. J. van Wees, Europhys. Lett. 7, 359 (1988).

${ }^{10}$ J. R. Hayes, A. F. J. Levi, and W. Wiegmann, Phys. Rev. Lett. 
54, 1570 (1985); J. R. Heiblum, M. I. Nathan, D. C. Thomas, and C. M. Knoedler, 55, 2200 (1985).

${ }^{11}$ A. Palevski, M. Heiblum, C. P. Umbach, C. M. Knoedler, A. M. Broers, and R. H. Koch, Phys. Rev. Lett. 62, 1776 (1989).

${ }^{12}$ P. C. van Son, H. van Kempen, and P. Wyder, J. Phys. F 17, 1471 (1987); V. S. Tsoi, N. P. Tsoi, and S. E. Yakovlev, Zh. Eksp. Teor. Fiz. 95, 921 (1989) [Sov. Phys._JETP 68, 530 (1989)].

${ }^{13}$ P. C. van Son, H. van Kempen, and P. Wyder, Phys. Rev. Lett. 58, 1567 (1987).

${ }^{14}$ Yu. V. Sharvin and N. I. Bogatina, Zh. Eksp. Teor. Fiz. 56, 772 (1969) [Sov. Phys._JETP 29, 419 (1969)].

${ }^{15}$ For an order-of-magnitude estimate of the importance of the magnetic field $B_{\text {ind }}$ induced by the transport current in our case we set the current $I=1 \mu \mathrm{A}$ and the point-contact separation $L=1.5 \mu \mathrm{m}$, then $B_{\text {ind }} \approx \mu_{0} I / 2 \pi L \approx 10^{-7} \mathrm{~T}$. This is several orders of magnitude smaller than the typical focusing fields of $10^{-1} \mathrm{~T}$, and may be neglected.

${ }^{16}$ For all measurements presented here the reference region was $s$, which was connected to ground, hence a negative voltage implies injection from $i$ into $s$ of hot electrons with energy in excess of $E_{F}$, while a positive voltage corresponds to focusing of cool unoccupied electron states below $E_{F}$.

${ }^{17}$ L. P. Kouwenhoven, B. J. van Wees, C. J. P. M. Harmans, J. G. Williamson, H. van Houten, C. W. J. Beenakker, C. T. Foxon, and J. J. Harris, Phys. Rev. B 39, 8040 (1989).

${ }^{18}$ U. Sivan, M. Heiblum, and C. P. Umbach, Phys. Rev. Lett. 63, 992 (1989).

${ }^{19}$ For a recent discussion of the measurement of local voltages see R. Landauer, IBM J. Res. Develop. 32, 306 (1988); A. D. Stone and A. Szafer, ibid. 